\title{
FATORES PREDISPONENTES PARA O INSUCESSO DA PUNÇÃO INTRAVENOSA PERIFÉRICA EM CRIANÇAS ONCOLÓGICAS
}

\author{
Hérica Martins B. Carvalho ${ }^{1}$, Luciano Marques dos Santos ${ }^{2}$, Larissa Freitas \\ Cerqueira $^{3}$, Cleonara Sousa Gomes e Silva ${ }^{4}$. \\ ${ }^{1}$ Voluntária pevic, graduanda em Enfermagem, Universidade Estadual de Feira de Santana. Email: \\ martins.herica@ hotmail.com \\ ²Orientador, Departamento de Saúde, Universidade Estadual de Feira de Santana. Email: \\ lucmarxenfo@yahoo.com.br \\ 3Participante do NUDES, Departamento de Saúde, Universidade Estadual de Feira de Santana. Emaill: \\ cerqueirafreitaslarisa@gmail.com \\ ${ }^{4}$ Participante do NUDES, Departamento de Saúde, Universidade Estadual de Feira de Santana. Email: \\ cleosilvauefs@gmail.com
}

PALAVRAS CHAVE: Enfermagem Oncológica; Criança hospitalizada; Cateterismo periférico.

\section{INTRODUÇÃO:}

O câncer infantil era considerado uma doença aguda com mau prognóstico. Atualmente, apresenta grande possibilidade de cura, com potencial aumento de sobrevida em aproximadamente $70 \%$ dos casos (MONTEIRO et al., 2014).A administração de quimioterápicos por via intravenosa em ciclos repetidos é um dos pilares do tratamento de pacientes com câncer (VILLARÍN ; ARAÚJO 2014). Atualmente, a quimioterapia é, dentre as modalidades de tratamento, a que possui maior incidência de cura de muitos tumores incluindo os mais avançados e a que mais aumenta a sobrevida dos portadores de câncer (ANDRADE; SILVA 2007). Manuseio e administração intravenosa de agentes citostáticos para o tratamento de pacientes com câncer é uma técnica que, dada a sua complexidade, requer uma equipe qualificada, conhecedora de seus efeitos adversos e potencial de riscos (VILLARÍN; BELDA, 2014).A quimioterapia por via endovenosa resulta em certas complicações como infiltrações, flebites e extravasamento. Diante disso, segundo Negri et al. (2012) garantir o sucesso da terapia intravenosa (TIV) significava garantir o êxito da inserção do dispositivo intravascular periférico.Assim, ao tentar estabelecer o estado da arte nacional e internacional referente aos fatores predisponentes para o insucesso da PIP em crianças com câncer, observou-se ausência de publicações sobre a temática. Diante disso, questionou-se: que fatores relacionados à criança, a TIV prévia e da PIP atual predispõem o insucesso da inserção de cateteres periféricos em crianças oncológicas? Este estudo tem relevância profissional, teórica, e social. No que se refere o ponto de vista profissional, os resultados poderão fornecer fundamentos científicos para a prática 
clínica dos profissionais de saúde envolvidos no cuidado de crianças e adolescentes com câncer que estão em uso de quimioterápicos por via intravenosa. OBJETIVO: Verificar os fatores predisponentes para o insucesso da PIP realizada em crianças oncológicas hospitalizadas no Hospital Estadual da Criança em Feira de Santana na Bahia.

\section{METODOLOGIA:}

Tratou-se de um estudo quantitativo, transversal, vinculado à pesquisa intitulada "Segurança do paciente pediátrico e sua família: estudo das tecnologias e eventos adversos relacionados à terapia intravascular periférica". realizado no Hospital Estadual da Criança (HEC), no ambulatório e clínica oncológica. Com 394 crianças e adolescentes com 29 dias de vida a 16 anos de idade que realizaram punção venosa periférica. Os dados foram coletados no período de abril 2015 a março de 2016, através de fonte primária através da observação do procedimento realizado pelo profissional, onde serão observadas as condições da rede venosa, o método de punção utilizado e as características da criança como edema em membros e agitação e por fonte secundária através da utilização do prontuário de cada criança selecionada. Os dados da observação e coleta em prontuário foram transportados para o formulário. A análise foi feita através do programaStatistical Package for Social Science (SPSS) versão 22.0, utilizando estatística descritiva. Este estudo está vinculado à pesquisa intitulada "Segurança do paciente pediátrico e sua família: estudo das tecnologias e eventos adversos relacionados à terapia intravascular periférica", que conta com o apoio da Pró-Reitoria de Pesquisa e Pós-Graduação da Universidade Estadual de Feira de Santana, o qual foi aprovado sob o parecer de $\mathrm{n}^{\circ} 841.612$.

\section{RESULTADOS E DISCURSSÃO:}

CARACTERIZAÇÃO DAS PUNÇÕES CONFORME O SUCESSO OU INSUCESSO NO PROCEDIMENTO DA PIP

Um total de 394 PIP foram observadas durante o período de coleta em campo, com 6,7\% (29) das PIP mal sucedidas (insucesso) e 93,3\% (365) PIP bem sucedidas. Dentre os motivos para o insucesso, 46,4\% (13) foram decorrentes de transfixação da veia, 46,4\% (13) punção ineficaz, e 7,2\% (3) outros motivos como rede venosa de difícil acesso. O número de tentativas de punção intravenosa variou de 2 a 6 tentativas, com uma média de 3,20 $\pm 1,1$, sendo que das punções que resultaram em insucesso 42,3 ocorreram em duas tentativas (tabela 1). 
Tabela 1: Distribuição das características das crianças com câncer que obtiveram insucesso da punção intravenosa periférica em um Hospital pediátrico público do interior da Bahia. Feira de SantanaBA. 2015 e 2016.

\begin{tabular}{lcc}
\hline \multicolumn{1}{c}{ Variáveis } & N & \% \\
\hline Obtenção do acesso venoso & & \\
$\quad$ Sucesso & 365 & 93,3 \\
Insucesso & 29 & 6,7 \\
Motivo do insucesso & & \\
$\quad$ Transfixação da veia & 13 & 46,4 \\
$\quad$ Punção ineficaz & 13 & 46,4 \\
$\quad$ Outros & 3 & 7,2 \\
Número de tentativas da PIP* & & \\
2 tentativas & 11 & 42,3 \\
3 tentantivas & 9 & 34,6 \\
4 ou mais tentativas & 6 & 23,1 \\
\hline
\end{tabular}

FONTE: coleta de dados. Feira de Santana- Bahia, 2015/2016.

ASSOCIAÇÃO ENTRE AS CARACTERÍSTICAS DA CRIANÇA COM A OCORRÊNCIA DE INSUCESSO NA INSERÇÃO DO DISPOSITIVO INTRAVASCULAR PERIFÉRICO

Para o cruzamento das variáveis foram consideradas todas as punções estudadas, ou seja, aquelas em que ocorreram insucesso $(n=29)$ e às que ocorreram sucesso $(n=$ 365).Houve diferenças estatísticas significantes entre, condição nutricional $(p=0,00285)$ presença de edema $(p=0,003)$, tempo de hospitalização $(p=>0,001)$ (tabela 5). Crianças obesas apresentaram prevalência cinco vezes maior do que as eutróficas para a ocorrência de insucesso da PIP, igualmente como crianças com presença de edema apresentaram prevalência de cinco vezes maior do que as tinham ausência de edema, assim como crianças que ficaram internadas mais que sete dias apresentaram prevalência cinco vezes maior do que as ficaram internadas menos de sete dias.

Tabela 2: Associação entre as variáveis relacionadas às crianças que obtiveram insucesso em um Hospital pediátrica no interior da Bahia, Feira de Santana, no período de 2015 e 2016.

\begin{tabular}{|c|c|c|c|c|c|}
\hline \multirow[t]{2}{*}{ Características } & \multicolumn{5}{|c|}{ Insucesso na obtenção do acesso intravenoso periférico } \\
\hline & $\operatorname{Sim} \mathbf{n}(\%)$ & Não n (\%) & p-valor & $\mathbf{R P}$ & IC \\
\hline \multicolumn{6}{|l|}{ Idade } \\
\hline Menor que 83 meses & $24(9,8)$ & $221(90,2)$ & $5,63 * *$ & 2,91 & $1,13-7,48$ \\
\hline Maior que 84 meses & $5(3,4)$ & $144(96,6)$ & & & \\
\hline \multicolumn{6}{|l|}{ Sexo } \\
\hline Feminino & $13(6,7)$ & $182(93,3)$ & $0,273^{*}$ & 0,829 & 041167 \\
\hline Masculino & $16(8,0)$ & $183(92,0)$ & & & $0,41-1,0 /$ \\
\hline Raça/Cor & & & & 1,51 & $0,74-3,08$ \\
\hline Branca/vermelha & $12(9,6)$ & $113(90,4)$ & $1,24 * *$ & & \\
\hline Negra/Parda & $17(6,3)$ & $252(93,7)$ & & & \\
\hline \multicolumn{6}{|l|}{ Condição Nutricional } \\
\hline Eutrófica & $22(6,4)$ & $323(93,6)$ & $0,00285^{*}$ & 5,7 & \\
\hline Desnutrida & $3(7,9)$ & $35(92,1)$ & & & $2,36-13,15$ \\
\hline Obesa & $4(36,4)$ & $7(63,6)$ & & & \\
\hline \multicolumn{6}{|c|}{ Histórico de prematuridade } \\
\hline Sim & $1(2,5)$ & $39(97,5)$ & $0,181 * *$ & 0,316 & \\
\hline Não & $28(7,9)$ & $326(92,1)$ & & & $0,044-2,26$ \\
\hline
\end{tabular}




\begin{tabular}{|c|c|c|c|c|c|}
\hline \multicolumn{6}{|l|}{ Presença de edema } \\
\hline Sim & $5(33,3)$ & $10(66,7)$ & \multirow[t]{2}{*}{$0,003 * *$} & \multirow[t]{2}{*}{5,26} & \multirow[t]{2}{*}{$2,33-11,87$} \\
\hline Não & $24(6,3)$ & $355(93,7)$ & & & \\
\hline \multicolumn{6}{|l|}{ Hiperatividade da criança } \\
\hline Sim & $20(16)$ & $105(84)$ & \multirow[t]{2}{*}{$20 *$} & \multirow[t]{2}{*}{4,78} & \multirow[t]{2}{*}{$2,24-10,20$} \\
\hline Não & $9(3,3)$ & $206(96,7)$ & & & \\
\hline \multicolumn{6}{|l|}{ Tempo de hospitalização } \\
\hline Menor que 7 dias & $10(27)$ & $27(73)$ & \multirow[t]{2}{*}{$>0,001 *$} & \multirow[t]{2}{*}{5,07} & \multirow[t]{2}{*}{$2,55-10,09$} \\
\hline Maior igual que 7 dias & $19(5,3)$ & $338(94,7)$ & & & \\
\hline
\end{tabular}

FONTE: Coleta de dados, Feira de Santana - Bahia, 2015/2016.

*Qui-quadrado de Person

**Teste exato de Fisher

Não foram observadas diferenças entre os grupos insucesso e sucesso da PIP para as variáveis sexo, idade, histórico de prematuridade, raça/cor e hiperatividades da criança, condição nutricional . O insucesso foi mais frequente em crianças menores de 6 anos, 11 meses e 29 dias (9,8\%), do sexo masculino (8,0\%), de raça/cor branca/vermelha $(9,6 \%)$, obesas $(36,4 \%)$, com ausência história de prematuridade (7,9\%), hiperativas no momento da PIP (16,0\%), e com membros edemaciados $(33,3 \%)$.

Nesse estudo, ao analisar os fatores predisponentes para o insucesso da PIP em crianças com câncer, comparando-se ao grupo que obteve sucesso, nota-se que houve uma prevalência de insucesso na instituição estudada de 6,7\%. A prevalência do insucesso na instituição em estudo está dentro variabilidade relatada em pesquisas nacionais e internacionais recentes que apontam uma taxa entre 4,2\% a $47,8 \%$ (JACOBSON; WINSLOW, 2005; CHINNOCK; THORNTON; HENDEY, 2007; HESS, 2010; RIKER, et al., 2011; NEGRI et al., 2012; PETERSON et al., 2012; REIGART et al., 2012; AVELAR; PETERLINI; PEDREIRA, 2013). Constatou-se que, a faixa etária predominante para a ocorrência de insucesso da PIP foram em crianças de 29 dias de vida a 6 anos, 11 meses e 29 dias de idade ( 77,3). Yen, Riegert, Gorelick (2008) identificaram que à medida que se aumenta a faixa etária dos grupos, o risco diminui, isso significa que a chance se ter um insucesso com crianças mais velhas é menor do que em grupos de crianças mais novas.

\section{CONCLUSÕES}

Este estudo apresenta algumas limitações, dentre elas a não uniformidade quanto ao procedimento da PIP, não haver um único grupo responsável pela PIP, a amostra ser baseada na quantidade de PIP e não no número de crianças. Por se apresenta como um estudo transversal, e haver a necessidade de um estudo em conjunto da exposição a um determinado fator e seu efeito, em um mesmo tempo. Este estudo possibilitou verificar 
alguns fatores predisponentes para o insucesso na PIP em crianças com câncer hospitalizadas no Hospital Estadual da Criança em Feira de Santana na Bahia.

\section{REFERÊNCIAS}

ANDRADE, M; SILVA, S.R. Administração de quimioterápicos: uma proposta de protocolo de enfermagem. Rev. Bras. enferm. vol. 60. n. 3. Brasília, 2007. CHINNOCK, B.; THORNTON, S.; HENDEY, G.W. Predictors of success in nurse-performed ultrasound-guided cannulation. The Journal of Emergency Medicine, v. 33, n. 4, p. 401-405, 2007.

AVELAR, A. F. M.; PETERLINI, M. A. S.; PEDREIRA, M. L. G. Assertividade e tempo de permanência de cateteres intravenosos periféricos com inserção guiada por ultrassonografia em crianças e adolescentes. Rev Esc Enferm USP., v. 47, n. 3, p. 539-546, 2013.

HESS, H.A. A biomedical device to improve pediatric vascular access success. Pediatr Nurs. v.36, p.259-263, 2010.

JACOBSON, A. F.; WINSLOW, E. H. Variables influencing intravenous catheter insertion difficulty and failure: An analysis of 339 intravenous catheter insertions. Heart \& Lung. v.34 n.5 p.345-359, 2005.

MONTEIRO,A. C. M. et al. A atuação do enfermeiro junto à criança com câncer: cuidados paliativos. Revenferm UERJ v.22, n.6. Rio de Janeiro. 2014.

NEGRI, D. C. et al. Fatores predisponentes para insucesso da punção intravenosa periférica em crianças. Rev. Latino-Am. Enfermagem, v. 20, n. 6, p. 1072-1080. 2012.

PETERSON, K.A. et al. Does the use of an assistive device by nurses impact peripheral intravenous catheter insertion success in children? Journal of Pediatric Nursing. v.27,p.134143, 2012.

REIGART, J. R. et al. Peripheral Intravenous Access in Pediatric Inpatients. Clin Pediatr. v.51 n.5 p.468-472, 2012.

RIKER, M. W. et al. Validation and refinement of the Difficult Intravenous Access Score: a clinical prediction rule for identifying children with difficult intravenous access. Acad Emerg Med., v. 18, n. 11, p. 1129-1134. 2011.

VILLARÍN, A.J.L; ARAUJO, P.L. Administración de quimioterapia intravenosa el paciente oncológico. Volume 13, Issue 1, 2014. 